\title{
Re-examining the Paradox of Structure: A Child Health Network Perspective
}

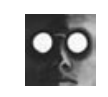

\author{
COMMENTARY
}

\section{Charmaine M. McPherson, RN, $\mathrm{PHD}(\mathrm{c})$}

St. Francis Xavier University

Founding Member and Steering Committee and Research Team Member

Child and Youth Health Networks of Canada (CYHNC)

Doctoral Candidate in Clinical Health Sciences, McMaster University

Past Director, Network for Children and Youth, Eastern Nova Scotia

\section{Janice K. Popp, MSW, RSW}

Director, Southern Alberta Child and Youth Health Network

Founding Member and Steering Committee and Research Team Member, CYHNC

Ronald R. Lindstrom, $\mathrm{PHD}, \mathrm{CHE}$

Child and Family Research Institute, BC

Founding Member and Steering Committee and Research Team Member, CYHNC

$\propto$

\section{ABSTRACT}

In their lead paper, Huerta, Casebeer and VanderPlaat argue that there are several key forces driving the development of health services delivery (HSD) networks, and propose a series of paradoxes and propositions to initiate this timely and essential dialogue. Ultimately, they submit that networks are likely to remain within the bealthcare system to build system capacity and drive integration. Given this, they challenge us to further the dialogue and investigate these networks. 
Re-examining the Paradox of Structure: A Child Health Network Perspective

While this peer commentary shares many of the lead authors' perspectives, the generic nature of the discussion does not bring us to the relative complexities revealed in some HSD network practices. A Canadian child health network lens is used to re-examine the lead paper's conceptualization of network typologies and the proposed paradox of structure. We combine network practice and academic expertise to highlight the structural, governance and leadership tensions between traditional bierarchical public service organizations and the non-hierarchical nature of interorganizational networks. Child health network leaders and members must examine and work with the challenges associated with importing traditional organizational cultures into an inter-organizationally networked context, while simultaneously maintaining these dual (or duelling) cultures.

THe LeAd PAPER by Huerta et al. offers many valuable insights regarding the challenges and opportunities inherent in health services delivery (HSD) networks. What is relatively unknown and unexplored at this point in the health and organizational management literature is the transferability or generalizability of empirical findings regarding these more generic HSD networks to inter-organizational child health networks (CHNs).

We respond to the lead paper from the perspective of a Canadian CHN, as a particular genre of inter-organizational HSD networks. We concur with the substantive content of the lead paper as a global current appraisal of HSD networks. Our response re-examines several of the concepts and issues raised by the authors and extends the discussion using a CHN lens. We re-examine the paradox of structure and key associated governance and leadership issues, and propose several propositions to guide further dialogue and inquiry.

\section{Perspective: CHNs - A Network Typology beyond Health Services Delivery}

Responding to the conceptualization of network types offered in Figure 1 of the lead paper, we argue that $\mathrm{CHN}_{\text {s }}$ are a blended form of conception, exploitation, exploration and implementation networks. With the exception of conception or exploration networks of the academic type, $\mathrm{CHN}$ serve all functions and fit the description of each outlined type concurrently under one umbrella network. We offer the following contextual overview to support this argument for a blended form of network conceptualization for CHNs.

$\mathrm{CHN}$ have developed as a promising means toward better health, social and educational service innovation and delivery; better policy formulation, enactment and enforcement; and, ultimately, better child and family health outcomes. These inter-organizational networks are emerging organizational structures in the 21 st century and have been proliferating in Canada over the past decade, with at least 20 such networks operating in five provinces (Popp et al. 2005), including the Southern Alberta Child and Youth Health Network (SACYHN) and the Network for Children and Youth, Eastern Nova Scotia (NCYENS). Some provincial governments have mandated the development of $\mathrm{CHN}$ as a means of service integration and child 
health outcome improvement (Province of Nova Scotia 1995), while other CHNs have developed voluntarily (SACYHN 2004).

$\mathrm{CHN}$ focus on the coordination of policy development, priority setting, service planning and service delivery in support of the health and well-being of children and their families within a provincial region. Fundamentally, in CHNs, as in other types of inter-organizational networks, organizations and stakeholders unite to reach goals that none of them can reach separately (Chisholm 1998).

These networks consist of complex inter-organizational partnerships formalized under a joint umbrella organization: the network. The membership of some CHNs can be diverse, including mental health services, school boards, child welfare services, provincial ministries, parents and community leaders, to name several. Some of the $\mathrm{CHN}$ members represent various service sectors, mirroring the service pattern usage of children and families with complex and multiple service needs. CHNs acknowledge the complexity of child health issues and the myriad of service options, or non-options, faced by this vulnerable population.

CHNs develop trans-disciplinary (Rosenfield 1992), cross-sectoral and publiccommunity partnerships that may span regional, provincial or national governmental boundaries. These complex partnerships are formalized within the CHN to support efforts concerning macro-issues such as child poverty, family violence and intersectoral and pan-governmental service planning and delivery (McPherson 2003).

Consistent with the $\mathrm{CHN}$ notion of public-community partnerships, in the lead paper Huerta, Casebeer and VanderPlaat reference the important shift to a more client- or patient-centred approach to clinical services delivery. In CHNs there is a growing understanding that the recipients of care also offer a valuable perspective that should inform program and policy development (Johnson 2000). However, this is difficult to enact in traditionally led, hierarchical organizations where decisions most often flow top down. In contrast, true inter-organizational networks are usually non-hierarchical in nature (Chisholm 1998) and strive to minimize the traditional power dynamics that tend to marginalize some players, especially family or community participants.

Although CHNs may deal with traditional disease-oriented service and policy issues, significantly, CHNs attempt to maximize the resources and perspectives of their diverse membership to tackle the multiple determinants of child, youth and family health and well-being, with a particular emphasis on health promotion, illness and injury prevention and early intervention. CHNs embrace the notion that positively impacting the health status of this population requires collaboration beyond the sole domain of what the public may see as the traditional health services sector.

\section{Paradox: Governance and Leadership Structures in CHNs}

Given the non-traditional nature of $\mathrm{CHN}$, especially in contrast to the public service sector, traditional governance structures and leadership styles do not necessarily work well. However, the alternative nonhierarchical approach alone does not seem to work either at all points in network development and evolution. We re-examine the lead paper's paradox of structure through a $\mathrm{CHN}$ lens.

Huerta, Casebeer and VanderPlaat noted that the culture of care and traditional organizational designs confound change efforts. We suggest that the matter is more 
complex than issues merely associated with traditional organizational designs. We believe that one of the most crucial tasks before us is to examine the intersection between traditional hierarchical organizational cultures, such as we find in most children's public service organizations, and the inter-organizational network culture. $\mathrm{CHN}$ members cross-exist within both domains and struggle to marry the two. $\mathrm{CHN}$ practitioners, leaders and members must examine and work with the challenges associated with importing organizational cultures into an inter-organizationally networked context, while simultaneously maintaining these dual (or duelling) cultures.

Traditional business-oriented interorganizational networks call for a loose coupling of network members or organizations and more informal organizational practices (Chisholm 1998). In CHNs, a tension between ideology and practice exists. Ideologically, the notion of non-hierarchical organizational structures, including governance, is appealing. However, in CHN practice, as is similarly seen in community health coalitions, members initially tend to formalize organizational processes, such as formal collaborative structures and governance procedures, emulating traditional public service sectors with their hierarchical arrangements with their respective histories, cultures and politics (Beery et al. 2005).

Beyond this attraction to and comfort or discomfort with traditional ways of organizing, we suggest that $\mathrm{CHN}$ members are learning to navigate the tension between traditional hierarchies back in their homebase organizations and the desired non-hierarchical arrangements of network structures. CHNs strive for a balance between organizational formalization and flexibility. For interorganizational CHN members - who may be staff in child welfare agencies, school boards and the like as well as concurrent $\mathrm{CHN}$ members - identifying, accepting and navigating this tension between the two structures is part of the network commitment.

$\mathrm{CHN}$ practice experiences in Nova Scotia and Alberta reveal that a more horizontal structure eventually emerges after the $\mathrm{CHN}$ has established itself. This horizontal structure of networks facilitates development of a leadership culture in which families and community members can play meaningful roles in policy, planning and service delivery. Networks can model and support the practice diffusion of this enhanced role and involvement of family and community in these central processes.

Significantly, despite provincial and federal legislations that tend to fragment and compartmentalize children's public services and leaders' thinking (Lindstrom 2000), $\mathrm{CHN}$ have been successful at regional and provincial levels in working collaboratively within inter-organizationally networked structures and cultures (NCYENS 1998; Dolinski 2005). However, there is a clear absence of any scholarly dialogue and empirical research exploring and explicating these issues to support $\mathrm{CHN}$ decision makers.

Network leadership is a second matter within the paradox of structure that requires further debate and dialogue. CHN leadership requires skill sets, processes and approaches that are generally not seen in traditional hierarchical public service sectors. Perhaps this is a seventh paradox of networks that is elemental to all six paradoxes outlined in the lead paper. Leadership styles must vary from the traditional organizational norm if a $\mathrm{CHN}$ is to achieve its fullest potential. Significantly, the leadership of a network, though perhaps supported by a network leader, is not the exclusive responsibility of that leader. Leadership of a network is a shared responsibility among 
all participants - a significant variation from traditional organizational models. The requisite $\mathrm{CHN}$ leadership style is different, and we submit that leadership style may be a key element that distinguishes networked organizations.

As indicated in the lead paper, networks are challenged by the question of how much and when structure is needed. This question is also crucial for $\mathrm{CHN}_{\mathrm{N}}$ since the need for structure versus leadership may sometimes be confused (Popp et al. 2005). As mentioned earlier, CHN members often seek structure early in development since it is familiar. Network leaders must respect and respond to members' desire to create formal structures, such as network agreements outlining roles, responsibilities and resource allocations. Concurrently, network leaders understand that formalized structure is the antithesis of that desired in early network development (Chisholm 1998). The leader's role is to protect the $\mathrm{CHN}$ from either the development of a comforting structure that will ultimately impede its creativity and responsiveness, or the creation of a premature structure that will send its tenuously engaged members fleeing as a consequence of the perceived demand for "too high a commitment too soon."

In developing and nurturing a $\mathrm{CHN}$, network leaders are required to "inculcate," as referenced in the lead paper, or educate members regarding network processes versus those of traditional organizations. $\mathrm{CHN}$ practice has also elucidated this experience. Keast et al. (2004) discussed a similar educational need within community coalitions. They noted that, although members are committed to collaboration, a lack of understanding of what it means to work in such structures early in development causes members to continue to construct traditional policies and management techniques characteristic of traditional public service sectors. They suggested that this created a tension that actually mitigated the positive attributes of such organizational arrangements.

Educating network members requires tactful, ongoing perturbing of traditional organizational thinking, such as structure equals security, accountability or evidence of action. The authors of the lead paper note that network decisions can be messy, time consuming and often frustrating. The flawed assumption here is that decisions in hierarchical organizations are not messy, time consuming or frustrating. In public services, such as healthcare and child welfare, practitioners are faced with the dual hierarchies: traditional organizational models and disciplinary-embedded hierarchies, such as those found in nursing or social work. This analysis does not often consider the more silent processes associated with critical issues such as gender, age, race, class and so on (McGibbon and McPherson 2005). Replete with structure, ancient histories, rules and cultures, these hierarchical paradigms hardly create quick, easy or accurate decision-making processes or even clear accountabilities.

From a practice perspective, $\mathrm{CHN}$ leaders may be ambivalent about structure because it takes steely resolve to just "let things evolve" as network experts have advised (Chisholm 1998). CHNs are under pressure to demonstrate early outcomes to solidify member commitment, gain credibility and maintain funding. So, if structure helps traditional stakeholders think the network is "delivering," then leaders may be tempted to adopt traditional structures and styles as well, even though the network literature and practice say this may not be in the CHN's best interests over the long term. 


\section{Propositions: Building CHN-Specific Literature}

This response addressed two key issues raised in the lead paper: (1) the proposed conceptualization of networks; and (2) the paradox of structure, particularly the tension between hierarchical and non-hierarchical structures and leadership styles.

There are major gaps in the health literature regarding inter-organizational CHNs. CHN practitioners and decision makers would benefit from further discussion and investigation into crucial, timely issues, such as $\mathrm{CHN}$ development, evolution and organizational effectiveness. To this end, we offer the following propositions to guide further $\mathrm{CHN}$ dialogue and inquiry:

1. CHNs are a complex blending of implementation, conceptual, exploratory and exploitative network types.

2. The tendency to develop traditional, hierarchical governance structures shifts to non-hierarchical models during $\mathrm{CHN}$ evolution as network members learn more about inter-organizational networks and recognize and develop a comfort level with the tension that exists between the two models.

3. CHN leaders require a different set of skills in addition to traditional human service-management skills. CHN leaders must be experts in both traditional as well as network organizational domains, and they must learn how to functionally and creatively blend the two worlds.

\section{Acknowledgement}

On behalf of the Child and Youth Health Networks of Canada organization, we acknowledge the contribution of the late Dr. Rupert Chisholm, Pennsylvania State University at Harrisburg, Pennsylvania, to our development. Before his passing in April 2004, Dr. Chisholm graciously spent some time with us in Baddeck, Nova Scotia, where he helped us further explicate and appreciate the complexities, advantages and timeliness of inter-organizational networks.

\section{References}

Beery, W., S. Senter, A. Cheadle, H.P. Greenwald, D. Pearson, R. Brousseau and G.D. Nelson. 2005.

"Evaluating the Legacy of Community Health Initiatives." American Journal of Evaluation 26(2): 150-65.

Chisholm, R.F. 1998. Developing Network Organizations: Learning from Theory and Practice. Reading, MA: Addison-Wesley.

Dolinski, C. 2005. Southern Alberta Child and Youth Health Network Evaluation Report. Calgary, AB: Southern Alberta Child and Youth Health Network.

Johnson, B.H. 2000. "Family-Centered Care: Four Decades of Progress. Families, Systems and Health." Journal of Collaborative Family Health Care 18(2): $137-56$.

Keast, R., M.P. Mandell, K. Brown and G. Woolcock. 2004. "Network Structures: Working Differently and Changing Expectations." Public Administration Review 64(3): 363-71.

Lindstrom, R.R. 2000. "Towards the Virtual Hospital: An Ecological Approach to Network Development in Health Services." Hospital Quarterly 3(3): 18-25.

McGibbon, E. and C. McPherson. 2005. "Interpretative Pedagogy in Action: Design and Delivery of a Violence and Health Workshop for Baccalaureate Nursing Students." Nursing Education 45(2): 81-5.

McPherson, C. 2003. Evaluating Child Health Networks: The Canadian Context. Paper presented at the World Child Health Congress, Vancouver, BC.

Network for Children and Youth, Eastern Nova Scotia. 1998. Eastern Region Child and Youth Services Project Evaluation. Sydney, NS: Author.

Popp, J.K., L.N. L’Heureux, C.M. Dolinski et al. 2005. "How Do You Evaluate a Network? A Canadian Child and Youth Health Network Experience." Canadian Journal of Program Evaluation 20(3): 123-50.

Province of Nova Scotia. 1995. Interdepartmental Agreement: Eastern Region Child and Youth Services Project. Halifax, NS: Author. 
Rosenfield, P.L. 1992. "The Potential of

Transdisciplinary Research for Sustaining and

Extending Linkages between the Health and Social

Sciences." Social Science and Medicine 35: 1343-57.

Southern Alberta Child and Youth Health Network.

2004. Child and Youth Health Networks: A National

Survey. Calgary, AB: Author.

\section{Longwoods eLetter

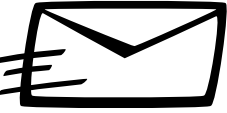

\section{Every Tuesday. Every Week.}

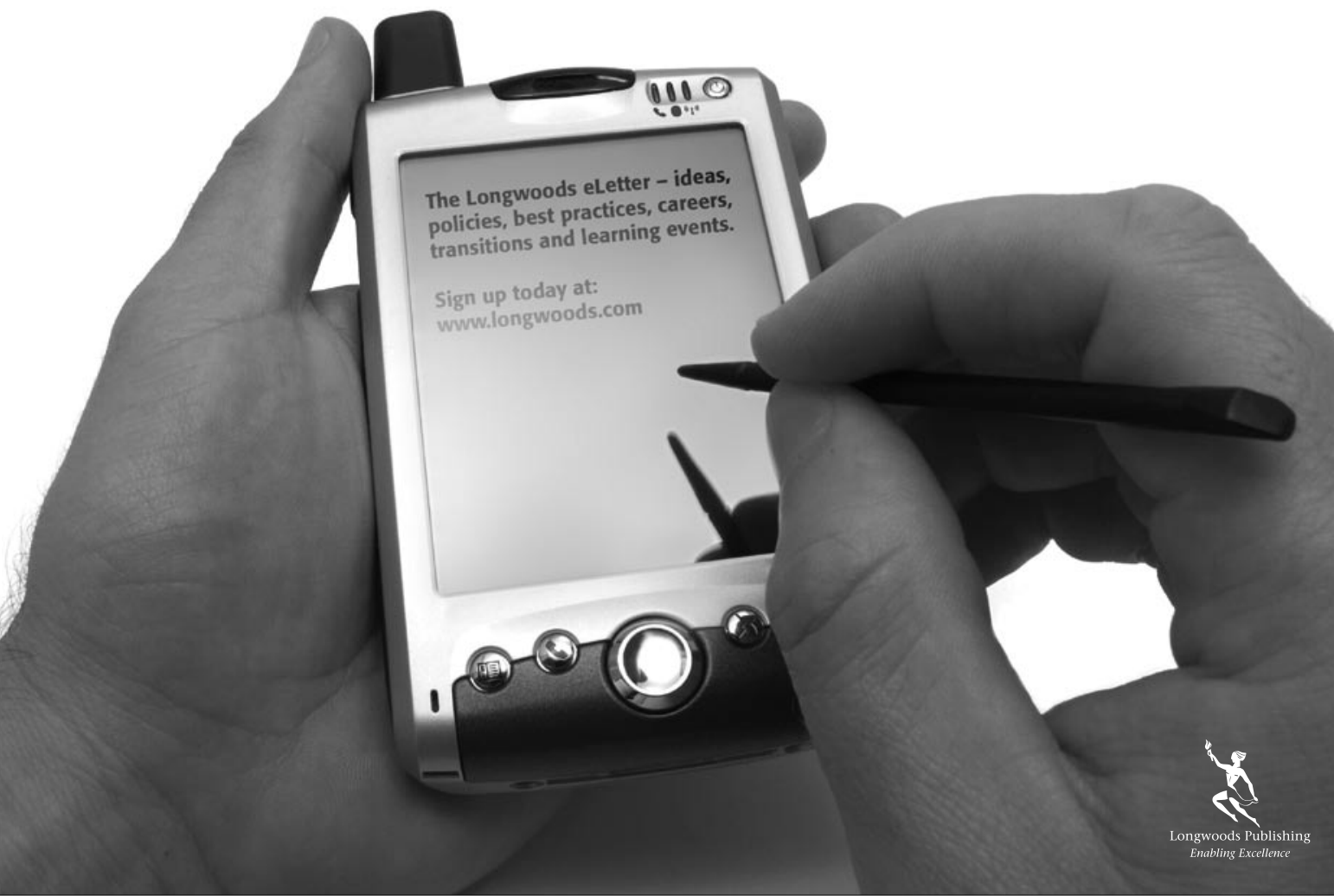

